\title{
Test observation results from the mid-infrared imager MIRTOS for the Subaru Telescope
}

\author{
Daigo Tomono, Yoshiyuki Doi, and Tetsuo Nishimura \\ Subaru Telescope, National Astronomical Observatory of Japan, \\ 650 North A'hoku Place, Hilo, Hawaii 96720, USA.
}

\begin{abstract}
MIRTOS, Mid-Infrared Test Observation System, is a high spatial resolution mid infrared (MIR) camera for the Subaru Telescope. It consists of two infrared imagers. One is for MIR bands with a Si:As array with $320 \times 240$ pixels. It has $21 \times 16$ arcsec field of view (FOV) with a pixel scale of 0.067 arcsec. It also images the pupil of the telescope. The other is a near infrared (NIR) camera. A $256 \times 256 \mathrm{InSb}$ array with 0.028 arcsec/pixel is used to image $7 \times 7$ arcsec FOV at one corner of the MIR FOV. We apply Shift-and-Add (SAA) technique; a technique that shifts images detecting the displacements and adds them to cancel seeing. However it is often difficult to shift and add MIR images using a reference within because of low sensitivity in MIR for short exposure time. We solve this problem utilizing NIR images taken simultaneously as position references. We call this method Two-Wavelength Shift-and-Add (TWSAA). In this paper we show results from the test observations. 1) Pupil image was taken. It shows hot structures around the secondary mirror that are now planned to be covered by reflecting plates to direct the beam to the sky. 2) Correlation of motion between MIR peak position and NIR centroid position shows that NIR images can be used as TWSAA reference for MIR observations. 3) On a standard star and the core NGC 1068, SAA method was applied to reconstruct images. Resulting images show higher spatial resolution than previous observations.
\end{abstract}

Keywords: MIR imaging, Two-Wavelengths Shift-and-Add, Subaru Telescope

\section{INTRODUCTION}

With the advanced large aperture telescopes, higher angular resolution are expected at all wavelengths including MIR (wavelengths of $10 \mu \mathrm{m}$ and $20 \mu \mathrm{m}$ ). Diffraction limited angular resolution for an $\mathrm{D}=8$-meter telescope at $\lambda=10 \mu \mathrm{m}$ is about $\lambda / \mathrm{D}=0.25$ arcsec. However, there are a couple of difficulties for observations in MIR with ground based telescopes. One of the difficulties is turbulence in the atmosphere. Another difficulty comes from thermal background emission.

The instantaneous image of an object, when observing from the ground, continuously dances around a center of long exposure image by seeing effects. It limits spatial resolution of imaging observations. The source of seeing can be divided into three; 1) mirror seeing, 2) dome seeing, and 3) natural atmospheric seeing. The first two are from turbulence induced around the telescope by temperature differences within the telescope and wind going through the telescope structure. Modern telescopes are designed to minimize these effects by cooling the telescope during the daytime and forcing a smooth air flow through the telescope during observations. Natural atmospheric seeing is made by patches of atmosphere where the beam from the source goes through. The patches have different refractive indexes because of different temperatures. Gradient of refractive index bends the direction of light coming through. The patches, moving with the wind, make the image dancing.

There are methods to compensate the atmospheric perturbation. One is called adaptive optics (AO) used mainly for observations in NIR and visible wavelengths. It is a method that detects the shape of perturbed wavefront and controls a deformable mirror to cancel the perturbation. As this method compensates wavefront deformation with a number of beam segments, AO is suitable to NIR and visible wavelength where the patches are smaller than MIR. The Subaru Telescope AO system has 36 beam segments to be compensated ${ }^{1]}$ Although it is applicable in almost

Further author information: (Send correspondence to D.T.)

D.T.: E-mail: tomono at subaru.naoj.org, Telephone: +1-808-934-7788, Fax: +1-808-934-5084.

Y.D.: E-mail: yoshi at subaru.naoj.org.

T.N.: E-mail: nishimra at @subaru.naoj.org. 


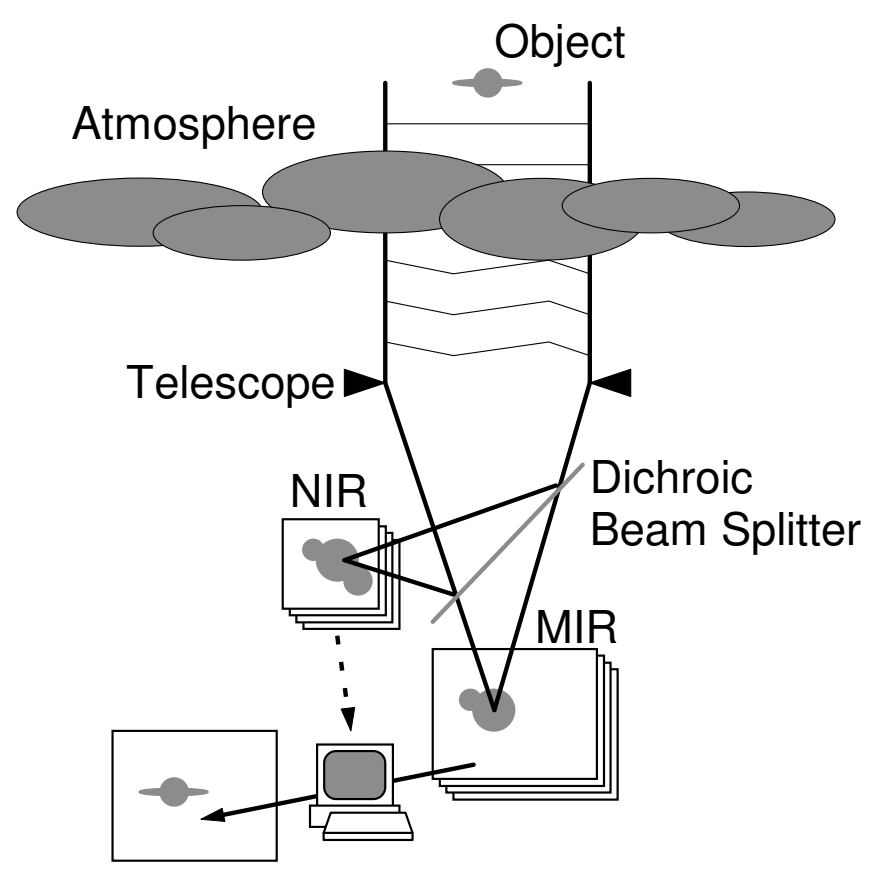

Figure 1. Concept of Two-Wavelength Shift-and-Add. Cameras take images simultaneously in two wavelengths. NIR images are used as a reference of atmospheric perturbation to shift and add the MIR image.

all astronomical applications, it has some disadvantages. First, it needs a source detectable in visible wavelength to measure the wavefront. Observation in visible wavelength suffers more interstellar and/or circumstellar extinction than observation in infrared does. Visible stars are often not available when observation is made toward regions such as star forming regions. Second, the system requires more mirrors at the room temperature that emits background thermal radiation.

For observations in MIR, simple Shift-and-Add (SAA) method might first appear to be good enough even with 8-meter telescopes because the patches are large enough. The SAA method adds many seeing-frozen images by shifting to cancel the image movements caused by the atmosphere. This method does not apply to MIR well because the reference source detection limit is much lower than in NIR or visible wavelength as a consequence of strong background radiation. Furthermore, sources of interest are rather extended in MIR. It makes it difficult to detect the image position movement precisely. To overcome these problems, we proposed to use simultaneous NIR images of a point source as a reference to shift and add MIR images.2] We call this method Two-Wavelength Shift-and-Add (TWSAA). Illustrated in Figure 1 is how it works. This takes the advantage that more point sources are available in NIR than in MIR, and NIR suffers less from extinction than in visible wavelengths. Note that this method also removes any other image movements, such as tracking errors of the telescope and/or beam positioning error of the chopping secondary.

The Subaru Telescope is not exclusively designed for infrared observations but is used for observations in other wavelengths. To check thermal emission coming from the telescope structure, entrance pupil of the telescope is mapped by MIRTOS. As Grundahl and Sorensen ${ }^{3}$ did, data will be used to reduce stray thermal emission into infrared instruments. In Table 1 basic specifications of MIRTOS are shown.

\section{THE INSTRUMENT}

The MIRTOS has two sets of camera optics for MIR and NIR. The optics shares the same field stop so that relative position of the images can be determined using pinholes that can be inserted in place for the field stop. Beam splitter divides MIR light and NIR light. The beam splitter and a flat mirror can be moved to align the axes of both of optics with the telescope optical axis. Entrance window is made of uncoated CdTe to allow a wide range of wavelength from NIR to $20 \mu \mathrm{m}$ into the dewars. The NIR dewar has the entrance window, the movable flat mirror 
Table 1. Basic parameters of MIRTOS

\begin{tabular}{|c|c|c|}
\hline & NIR & MIR \\
\hline \multirow[t]{2}{*}{$\overline{\mathrm{FPA}}$} & $\mathrm{SBRC}$ InSb & SBRC Si:AS IBC \\
\hline & $256 \times 256$ pixels & $320 \times 240$ pixels \\
\hline Pixel scale & $\lambda / 2 \mathrm{D}(2.2 \mu \mathrm{m} 8.2 \mathrm{~m})=0.028^{\prime \prime}$ & $\lambda / 3 \mathrm{D}(8 \mu \mathrm{m} 8.2 \mathrm{~m})=0.067^{\prime \prime}$ \\
\hline Field of View & $7.1 " \times 7.1 " a$ & $21.5 " \times 16.1 "$ \\
\hline Option & & Pupil imaging mode \\
\hline Filters & $\mathrm{J}, \mathrm{H}$, and $\mathrm{K}^{\prime}$ & various $\lambda_{c}$ with $\Delta \lambda \sim 1 \mu \mathrm{m}$ in N-band, and $18.5 \mu \mathrm{m}$ \\
\hline Limiting flux $b$ & 4.8 Jy at $\mathrm{K}^{\prime}$ for TWSAA ref $(0.1+0.1 \mathrm{sec})$ & $\begin{array}{l}0.5 \mathrm{Jy} \text { at } 10 \mu \mathrm{m} \\
2.5 \mathrm{Jy} \text { at } 20 \mu \mathrm{m}\end{array}$ as TWSAA result $(12+12 \mathrm{sec})$ \\
\hline
\end{tabular}

${ }^{a}$ NIR FOV is located at a corner of MIR FOV.

$b$ Typical snap-shot observation with $5 \sigma$ detection limit.

and the beam splitter. While NIR light is reflected by the beam splitter into the dewar, MIR light goes through to the MIR dewar attached to the side of NIR dear. The two dewars shares the same vacuum so that there is no window between the dewars. The arrays and optics are cooled by two closed cycle coolers. Both dewar has a set of read out electronics that are connected to two host computers. Data are processed on the host computers and are sent to the telescope system. Figure 2 shows MIRTOS attached to the telescope with other instruments in Cassegrain automated instrument exchanger CIAX-3.4 Details of designs of the MIRTOS are described in Ref. 2.

\section{PUPIL IMAGE OF THE TELESCOPE}

With a pupil imaging lens inserted using one position of the filters installed in the camera section, MIRTOS can obtain entrance pupil image. The field of view (FOV) in this mode is limited by the Lyot stop that reduces light coming from outside of the secondary mirror. However, the vicinity of the secondary mirror can be imaged by moving the beam adjustment movable flat mirror. In Figure 3, mosaiced image of the entrance pupil of the telescope is shown. This image was taken by using an $8.9 \mu \mathrm{m}$ filter on the night of August 25, 1999. Dark images taken with the cold shutter closed were subtracted. With the telescope looking at the zenith at the stow position, the top screen is seen as a crescent shaped hot area on the secondary mirror. We have found that the bases of spiders attached to the secondary mirror are bright in MIR. They might be excess background sources if Lyot stop of an instrument is oversized compared to the secondary mirror image on it. Tilted reflectors are planned to be attached on the spider bases to direct the beam to the sky. On the secondary mirror, the four spiders are seen. These spiders are weaker than the ones outside the secondary mirror. Mirrors are already attached on the spiders so that the instruments do not see the hot spiders. Also we can see center cone in the center of the secondary mirror.

\section{CORRELATION OF IMAGE MOTION BETWEEN MIR AND NIR}

In this section, correlation of image motion in MIR and NIR will be discussed. On January 17, 2000, the asteroid (1) Ceres was observed simultaneously in MIR and NIR with frame interval of 0.032 sec and 0.096 seconds respectively. After 3 seconds of integration, an offset was made on the telescope pointing to take background image on adjacent sky. Two sets of 96 images in MIR and 32 images in NIR are accumulated for a nodding cycle. A background image was made averaging the 96 or 32 images and subtracted from each on-source image. After the subtraction of the nodding pair, Airy pattern was convolved to reduce noise in measuring peak position. Location of pixel with maximum value after convolution is defined to be the peak position. Centroid position was also measured in the same manner with Gaussian with a bigger size (0.8" FWHM for NIR, 0.7" FWHM for MIR) applied in convolution.

Figure 4 shows measured image motions on K'-band and $12.3 \mu \mathrm{m}$ image on the left panels. Upper two panels are for $\mathrm{K}^{\prime}$ movement that we see smoother movement on centroid than on peak. Bottom panel shows peak movement on $12.3 \mu \mathrm{m}$ that resembles to $\mathrm{K}^{\prime}$ centroid movement.

In the right panel in Figure 4 correlation factors between $K^{\prime}$ and three different wavelength in MIR are shown. To take correlation, interpolated or extrapolated position was located on a straight line connecting the peak and the centroid on NIR images. Abscissa corresponds to the line. At the long tick mark labeled as K' Peak, correlation factor between $K^{\prime}$ peak and MIR peak is calculated, while correlation factor between K' centroid and MIR peak is 


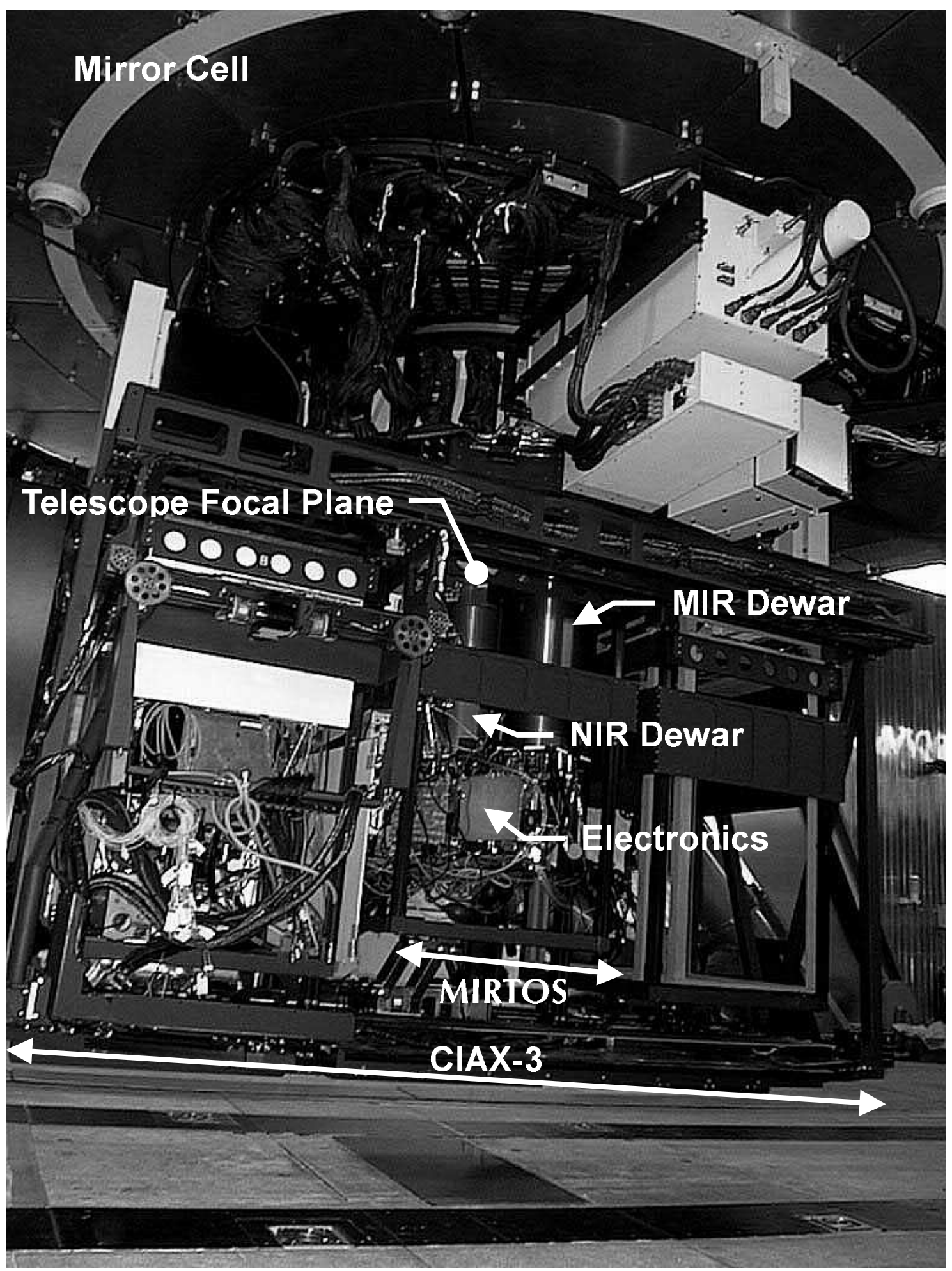

Figure 2. MIRTOS attached on the telescope. MIRTOS is mounted in one of three slots of Cassegrain automated instrument exchanger CIAX-3.4] Back of the primary mirror cell of the telescope is seen at the top of the photograph. In the center is MIRTOS with other instruments at the sides. There are two dewars each for the MIR camera and NIR camera. Array drive electronics are also seen. Host computers and other electronics are in the other side of the instrument. 


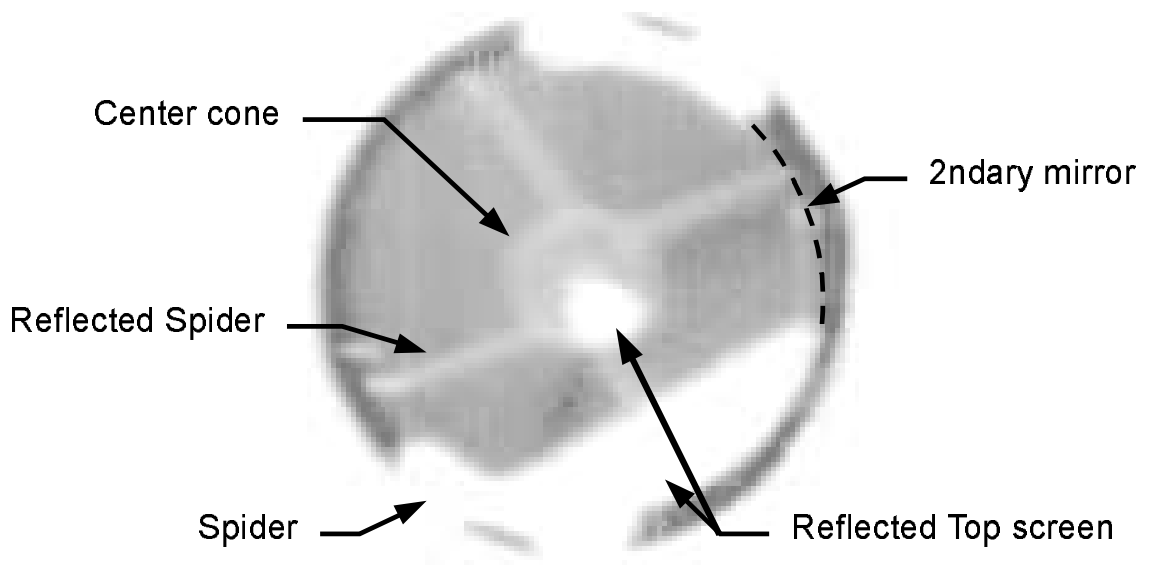

Figure 3. Pupil image of the telescope seen at $8.9 \mu \mathrm{m}$. Movable flat mirror in MIRTOS was used to mosaic images to see outside of the secondary mirror. Gray scale is logarithmic to enhance structure on the secondary mirror, with lighter area with brighter emission. The field is limited by a baffle around the tertiary mirror. The dark ring is the sky with the secondary mirror seen as a lighter disk inside. The dark ring of the sky is intercepted by bright spider structures attached to the secondary mirror. Crescent shaped area on the secondary mirror is a reflection of the telescope top screen. Also fainter spider structure and the center cone is seen. A bright spot in the center cone is a reflection from the top screen again. Spiders seen on the secondary mirror are darker than the one seen directly because they have reflecting plate on them so that they reflect light from the sky.

measured at the long tick mark labeled K' Centroid. The correlation factors peak at the centroid. This panel shows that we only have to detect centroid positions in NIR images to be used as position reference of MIR image in the Two-Wavelength Shift-and-Add (TWSAA) method.

\section{RECONSTRUCTION OF IMAGE USING SHIFT-AND-ADD TECHNIQUE}

\subsection{Point Source}

A sample Shift-and-Add result is shown in Figure 5 to demonstrate the angular resolution of the Subaru Telescope and the MIRTOS. $\beta$ And was observed with $11.7 \mu \mathrm{m}$ filter on January 17, 2000. Integration time for each single frame was 0.032 seconds. After taking 96 frames in 3 seconds, the telescope was offset to make a nodding pair. As we did on Ceres in Section 4 background was subtracted from the images. In the left panel of Figure 5 one of the 96 frames is shown. It is almost like the Airy pattern, however, the image is seen elongated in the direction from north-west (upper-right) to south-east (lower-left). Simply adding the 96 frames that is taken in a duration of 3.1 seconds makes the image shown at the middle of the Figure. Its FWHM is 0.34 arcsec. We can see a hint of diffraction ring on the image but it is broader than it should be. Shift-and-Add (SAA) method was applied on the image shown in the right most panel of the Figure 5 . We clearly see the diffraction ring around the star. FWHM of the image is $0.20 \mathrm{arcsec}, 10 \%$ more than that of the Airy pattern. Strehl ratio was also increased from 0.23 for normal integration to 0.45 after SAA.

\subsection{NGC 1068}

NGC 1068 is a prototype of Seyfert 2 galaxies. It has an Active Galactic Nuclei (AGN) in the center of the galaxy with a suspected optically opaque torus that obscures the light from the AGN as described in e.g. Ref. [5] Ultraviolet imaging polarimetry with Hubble Space Telescope revealed a centro-symmetric polarization pattern ${ }^{6}$ having a center where radio emission has an emission component.7 A MIR emission peak was detected at the position by Braatz et al[8. Bock et a ${ }^{[9]}$ resolved the MIR emission structure at the northeast by using the Hale $5 \mathrm{~m}$ telescope after deconvolution. Their deconvolved images also show an elongation to the south of the central peak.

The central structure of the NGC 1068 AGN was observed on the night of January 17, 2000. Nodding pairs are obtained in the same way as for $\beta$ And. Subsequent processes were also done as $\beta$ And to obtain Shift-and-Add 

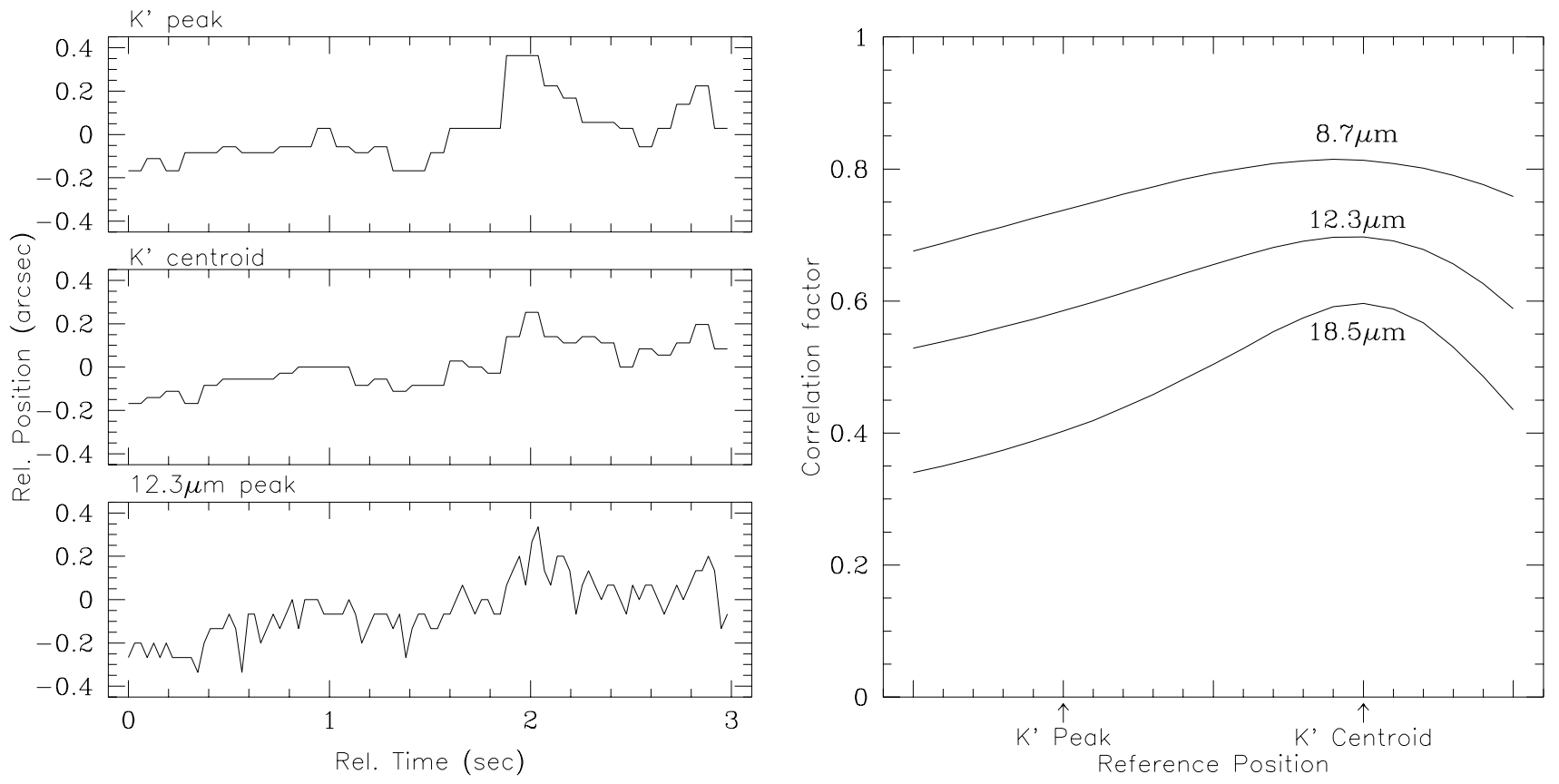

Figure 4. Correlation of simultaneous image motion in MIR and NIR. In the left panel is time sequence of image motion measured simultaneously in K'-band and $12.3 \mu \mathrm{m}$ for the asteroid (1) Ceres. On the left panel, time sequence of image positions are plotted. From the top to the bottom panels, movement of K' peak, K' centroid, and $12.3 \mu \mathrm{m}$ centroid are plotted. Frame interval is 0.032 seconds for MIR and 0.096 seconds for K'. Plotted in the right panel are correlation factors of the image motion between K'-band and various MIR wavelengths. Abscissa corresponds to K'-band reference position interpolated or extrapolated on a straight line connecting its peak and centroid. It shows that the peak positions in MIR images moves along with K' centroid positions rather than K' peak positions.

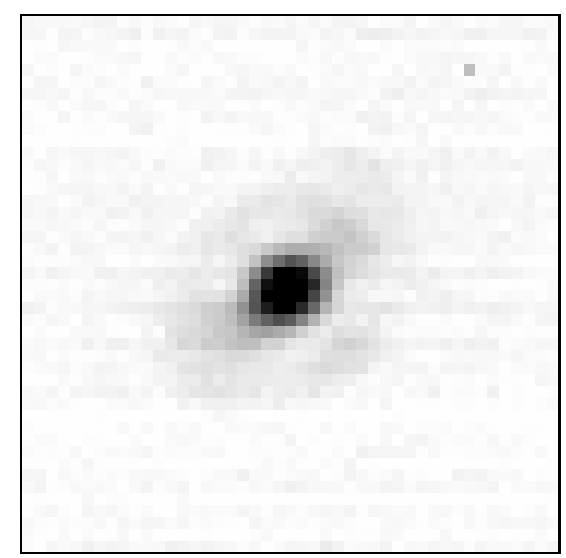

Single integration $0.032 \mathrm{sec}$

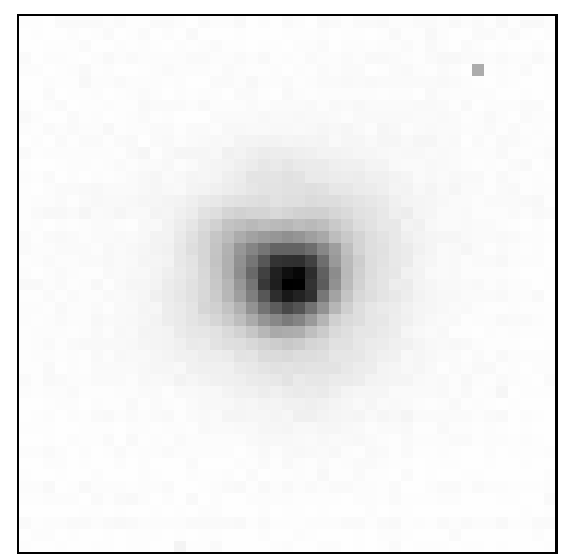

Normal integration for $3.1 \mathrm{sec}$

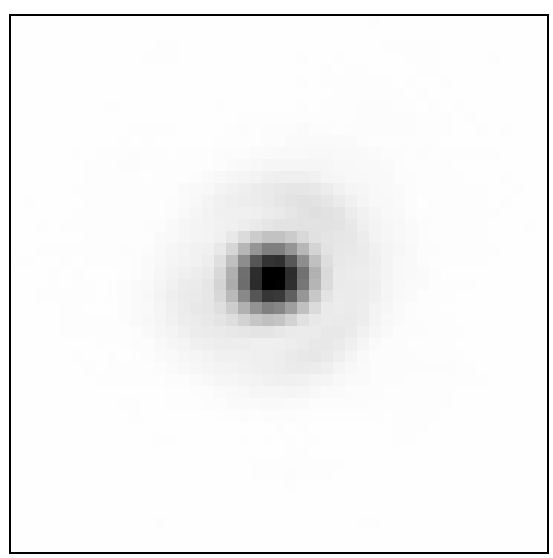

Shift-and-Add for $3.1 \mathrm{sec}$

Figure 5. Short exposure image, simply added image without Shift-and-Add, and Shift-and-Add image of $\beta$ And in $11.7 \mu \mathrm{m}$. Squares are $3 \times 3$ arcsec with north up and east to the left. One of the single frames in $11.7 \mu \mathrm{m}$ is shown in the left panel with the sky subtracted by a nodding pair. In the middle is a simple addition of 96 single frames taken in a duration of 3.1 seconds $(F W H M=0.34$ arcsec $)$. Shift-and-Add method reveals the diffraction ring of the star in the right panel $(\mathrm{FWHM}=0.20 \mathrm{arcsec})$ with the same frames used for the image in the middle. 


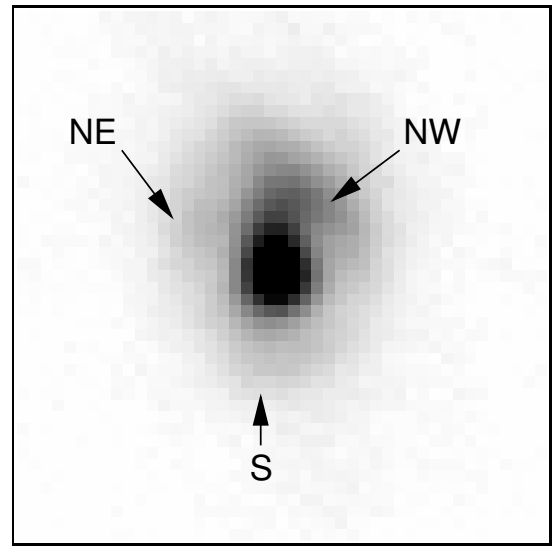

$11.7 \mu \mathrm{m}$

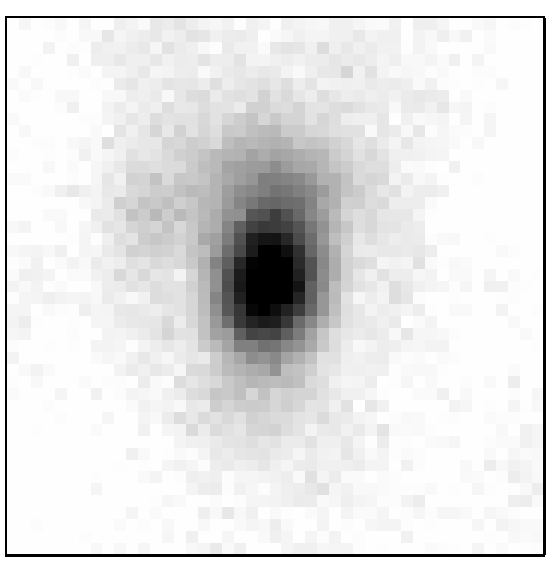

$18.5 \mu \mathrm{m}$

Figure 6. Shift-and-Add image of a Seyfert 2 galaxy NGC 1068. Squares are $3 \times 3$ arcsec with north up and east to the left. On both of the images, 384 frames of 0.032 seconds integration are combined to make total on-source integration time of 12 seconds. Marked on $11.7 \mu \mathrm{m}$ image are extended structure like the flag of an eighth musical note (NW) and locations of slight hints of point-like emission (S and NE).

images. Since the nucleus of NGC 1068 is bright and compact enough in MIR for simple Shift-and-Add method, TWSAA is not used. In Figure [6] resultant images before deconvolution are shown. As extensions of emission of lower level in $11.7 \mu \mathrm{m}$, we can see slight hints of emission components marked as S and NE. These components are in the same direction from the central peak as the structure resolved in Ref. 9] Moreover, an extended structure that has not been detected is seen in the north-west of the central peak extending from north-east to south-west. It is marked as NW in Figure 6 of which shape looks like the flag of an eighth musical note with the head at the central peak. We can see an elongation of this component also in the $18.5 \mu \mathrm{m}$ image. Further reduction of the data, including deconvolution, reveals the structure of the emission components and will be discussed eleswhere!10

\section{CONCLUSION}

We made a high spatial resolution MIR camera, MIRTOS. It also has a NIR camera that takes NIR images simultaneously at the same FOV. The combination of the two cameras enables us to apply TWSAA method to reconstruct images even without a reference in MIR images. Also, the MIR camera images the entrance pupil of the telescope to check foreground emission sources from the telescope structure. We conducted test observations with the instrument. Pupil image of the telescope showed that the bases of spiders attached to the secondary mirror can be stray emission sources. With simultaneous observation in MIR and NIR of Ceres, correlation between positions of the image was good even between $18.5 \mu \mathrm{m}$ and K'-band. Strehl ratio for a $11.7 \mu \mathrm{m}$ image of $\beta$ And made better to 0.45 by SAA from 0.23 for normal integration. For NGC 1068, SAA images in $11.7 \mu \mathrm{m}$ showed emission structures that has not been detected in former observations.

\section{ACKNOWLEDGMENTS}

We appreciate Dr. Hirokazu Kataza of University of Tokyo for providing us with the design of array drive/readout electronics. Mr. Norio Okada and staffs in the Machine Shop in Advanced Technology Center of National Astronomical Observatory of Japan, Tokyo made precise parts for array cassettes. Mr. Eliott Solheid and the staff of Infrared Laboratories Inc. in Tucson, Arizona fabricated the dewars and optics therein. Light and precise dewar mounting structure was made by Mr. Kerry Martin of Specialized Tool \& Machine in Hawi, Hawaii. We are also thankful to all the staff of the Subaru Telescope including Dr. Tomonori Usuda and Dr. George Kosugi who supported us for observations, Mr. Brian Elms who supported us with his machine shop, and Dr. Tetsuharu Fuse for providing us with the position of Ceres. DT was supported for a period of design and construction of the instrument by a Research Fellowship for Young Scientists from Japan Society for the Promotion of Science. 


\section{REFERENCES}

1. H. Takami, N. Takato, M. Otsubo, T. Kanzawa, Y. Kamata, K. Nakashima, and M. Iye, "Adaptive optics system for Cassegrain focus of Subaru 8.2-m telescope," in Adaptive Optical System Technologies, D. Bonaccini and R. K. Tyson, ed., Proc. SPIE 3353, pp. 500-507, 1998.

2. D. Tomono and T. Nishimura, "Mid infrared test observation system (MIRTOS) for SUBARU," in Infrared Astronomical Instrumentation, A. M. Fowler, ed., Proc. SPIE 3354, pp. 1150-1157, 1998.

3. F. Grundahl and A. N. S $\phi$ rensen, "Detection of scattered light in telescopes," AESApS, 116, pp. 367-371, 1996.

4. T. Usuda, K. Omata, M. Yutani, T. Noguchi, and T. Nishimura, "CIAX: Cassegrain instrument auto exchanger for the Subaru Telescope," in Advanced Telescope and Instrumentation Control Software, H. Lewis, ed., Proc. SPIE 4009, in press.

5. R. Antonucci, "Unified models for active galactic nuclei and quasars," ARAA, 93, pp. 473-521, 1993.

6. A. Capetti, F. Macchetto, D. J. Axon, W. B. Sparks, and A. Boksenberg, "Hubble Space Telescope imaging polarimetry of the inner nuclear region of NGC 1068," ApJ, 452, pp. L87-L89, 1995.

7. J. F. Gallimore, S. A. Baum, and C. P. O'Dea, "The subarcsecond radio structure in NGC 1068. II. implications for the central engine and unifying schemes," ApJ, 464, pp. 198-211, 1996.

8. J. A. Braatz, A. S. Wilson, D. Y. Gezari, F. Varosi, and C. A. Beichman, "High-resolution mid-infrared imaging and astrometry of the nucleus of the Seyfert galaxy NGC 1068," ApJ, 409, pp. L5-L8, 1993.

9. J. J. Bock, K. A. Marsh, M. E. Ressler, and M. W. Werner, "High-resolution mid-infrared imaging of the nucleus of NGC 1068," ApJ, 504, pp. L5-L10, 1998.

10. D. Tomono, "A new mid-infrared camera MIRTOS for the Subaru Telescope using Two-Wavelength Shift-andAdd Technique," PhD thesis, University of Tokyo, in preparation. 